\title{
АНАЛИЗ ВЛИЯНИЯ ЛИНЕЙНО ВОЗРАСТАЮЩЕГО ВДУВА И ЛИНЕЙНО ВОЗРАСТАЮЩЕГО ТЕМПЕРАТУРНОГО ФАКТОРА НА ПАРАМЕТРЫ МАТЕМАТИЧЕСКОЙ МОДЕЛИ И ЛОКАЛЬНЫЕ ХАРАКТЕРИСТИКИ ТЕПЛОМАССООБМЕНА И ТРЕНИЯ НА ПРОНИЦАЕМЫХ ПОВЕРХНОСТЯХ ГЛА
}

\author{
Г. Г. Бильченко, Н. Г. Бильченко \\ Казанский национальный исследовательский технический университет \\ (КНИТУ - КАИ) им. А. Н. Туполева
}

Поступила в редакцию 10.09.2019 г.

\begin{abstract}
Аннотация. Исследуются свойства математической модели управления тепломассообменом и трением в ламинарном пограничном слое на проницаемых цилиндрических и сферических поверхностях гиперзвуковых летательных аппаратов. Рассмотрен случай применения на всём участке управления сочетания линейно возрастающего вдува, линейно возрастающего температурного фактора и постоянного магнитного поля. Получены зависимости параметров математической модели и локальных характеристик тепломассообмена и трения от управляющих воздействий. Приведены результаты вычислительных экспериментов.

Ключевые слова: управление, тепломассообмен, ламинарный пограничный слой, гиперзвуковые течения, проницаемые поверхности, линейно возрастающий вдув, линейно возрастающий температурный фактор, параметры математической модели, локальные характеристики тепломассообмена и трения.
\end{abstract}

\section{ВВЕДЕНИЕ}

Данная работа является расширенным вариантом материалов докладов, представленных на LXXII научную конференцию «Некоторые актуальные проблемы современной математики и математического образования. Герценовские чтения-2019» [1] и XII Международную научную конференцию «Современные методы прикладной математики, теории управления и компьютерных технологий» (ПМТУКТ-2019) [2].

C помощью метода обобщённых интегральных соотношений А. А. Дородницына [3] для систем дифференциальных уравнений в частных производных (ДУЧП), описывающих ламинарные пограничные слои (ЛПС), на участке управления для проницаемых цилиндрических и сферических поверхностей гиперзвуковых летательных аппаратов (ГЛА) в $[4,5]$ получены аппроксимирующие системы обыкновенных дифференциальных уравнений (ОДУ).

В данной работе, продолжающей исследование свойств математической модели ЛПС электропроводящего газа на проницаемых цилиндрических и сферических поверхностях ГЛА [4-9], рассматривается влияние (на всём участке управления [10]) следующего сочетания управляющих воздействий: линейно возрастающего вдува, линейно возрастающего температурного фактора и постоянного магнитного поля:

1) на параметры математической модели ЛПС $\theta_{0}(x), \theta_{1}(x), \omega_{0}(x), \omega_{1}(x)$;

2) на локальные характеристики тепломассообмена и трения $q(x), f(x)$.

Являясь продолжением $[8,9]$, данная статья сохраняет введённые в них обозначения.

(c) Бильченко Г. Г., Бильченко Н. Г., 2019 


\section{Г. Г. Бильченко, Н. Г. Бильченко}

\section{1. ПОСТАНОВКА ЗАДАЧИ}

Рассмотрим следующую прямую задачу [7]:

$$
\left(m, \tau_{w}, s\right) \rightarrow(q, f, \eta ; Q, F, N) .
$$

По заданным управлениям: $m(x)-в д у в y$ в ЛПС, где $x \in X=[0 ; 1]$, а ось $x$ направлена вдоль контура тела; $\tau_{w}(x)=T_{w}(x) / T_{e_{0}}-$ температурному бактору, где $T_{w}(x)$ - температура стенки, а $T_{e_{0}}$ - температура в точке торможения $x_{0}=0$ потока; $s(x)=\sigma B_{0}^{2}(x)$ - магнитному полю требуется рассчитать параметры $\theta_{0}\left(x ; m, \tau_{w}, s\right), \theta_{1}(\ldots), \omega_{0}(\ldots), \omega_{1}(\ldots)$ математической модели ЛПС $[5,7]$ для случаев обтекания боковой поверхности кругового цилиндра и поверхности сферического носка. Для нахождения параметров $\theta_{0}, \ldots, \omega_{1}$ ЛПС применяется объединённая аппроксимирующая система ОДУ (5)-(8) [7] с начальными условиями, полученными из объединённой нелинейной алгебраической системы (10)(13) [7]. После этого определяются локальный тепловой поток $q\left(x ; m, \tau_{w}, s\right)$; локальное напряжение трения $f\left(x ; m, \tau_{w}, s\right)$; локальная мощность системы, обеспечивающей вдув $\eta\left(x ; m, \tau_{w}, s\right)$; интегральные характеристики $Q, F, N$.

Пусть фиксированы значения неизменяемых параметров:

$$
\begin{gathered}
\text { число Маха } M_{\infty} \in[10 ; 40], \\
\text { высота полёта } H \in[10 ; 30][\kappa \mathcal{~}], \\
\text { радиус тела } R \in[0,1 ; 1][\mathcal{M}] .
\end{gathered}
$$

Пусть диапазоны изменения управляющих параметров ограничены:

$$
\begin{gathered}
m \in M^{c}=[0 ; 1], \\
\tau_{w} \in T^{c}=[0,15 ; 0,9], \\
\left.s \in S^{c}=\left[0 ; 5 \cdot 10^{4}\right][\text { Tл/(OM } \cdot \mathcal{M})\right] .
\end{gathered}
$$

Далее индекс “ $w$ " параметра $\tau_{w}$ и размерность $[$ Тл/(OM $\cdot \mathcal{M})]$ параметра $s$ опущены.

Линейный вдув $m(x)$, определяемый законом (8) [8]

$$
\begin{gathered}
m(x)=m\left(x ; m_{0}, m_{1}\right)= \\
=m_{0} \cdot(1-x)+m_{1} \cdot x=m_{0}+m^{\prime} \cdot x,
\end{gathered}
$$

где $m_{0}, m_{1} \in M^{c}, m^{\prime}=m_{1}-m_{0}$, назовём [1] возрастающим (для $m^{\prime}>0$ ) или убьвающим (для $\left.m^{\prime}<0\right)$ слабо при $\left|m^{\prime}\right| \in(0 ; 0,3)$, умеренно при $\left|m^{\prime}\right| \in[0,3 ; 0,7)$, сильно при $\left|m^{\prime}\right| \in[0,7 ; 1]$.
Линейный температурный фактор $\tau(x)$, определяемый законом(8) [9]

$$
\begin{gathered}
\tau(x)=\tau\left(x ; \tau_{0}, \tau_{1}\right)= \\
=\tau_{0} \cdot(1-x)+\tau_{1} \cdot x=\tau_{0}+\tau^{\prime} \cdot x,
\end{gathered}
$$

где $\tau_{0}, \tau_{1} \in T^{c}, \tau^{\prime}=\tau_{1}-\tau_{0}$, назовём [1] возрастающим (для $\tau^{\prime}>0$ ) или убьвающим (для $\left.\tau^{\prime}<0\right)$ слабо при $\left|\tau^{\prime}\right| \in(0 ; 0,25)$, умеренно при $\left|\tau^{\prime}\right| \in[0,25 ; 0,5)$, сильно при $\left|\tau^{\prime}\right| \in[0,5 ; 0,75]$.

Обозначим

$$
\begin{gathered}
M_{05}^{d}=\{0 ; 0,05 ; \ldots ; 1\} \subset M^{c}, \\
M_{25}^{d}=\{0 ; 0,25 ; 0,5 ; 0,75 ; 1\} \subset M_{05}^{d}, \\
M_{25^{\prime}}^{d}=\{0,25 ; 0,5 ; 0,75\} \subset M_{25}^{d}, \\
T_{05}^{d}=\{0,15 ; 0,2 ; \ldots ; 0,9\} \subset T^{c}, \\
T_{15}^{d}=\{0,15 ; 0,3 ; \ldots ; 0,9\} \subset T_{05}^{d}, \\
T_{15^{\prime}}^{d}=\{0,15 ; 0,45 ; 0,6\} \subset T_{15}^{d} .
\end{gathered}
$$

Вычислительные эксперименты для удобства сравнения с $[4,6,8,9]$ выполнены для воздуха в атмосфере Земли при $H=10[\kappa \mathcal{~}]$, $M_{\infty}=10, R=0,1[\mathcal{M}]$.

На левой половине рис. 1 представлены линейно возрастающие $\left(m_{0}<m_{1}\right)$ вдувы $(8)$ с $m_{0}, m_{1} \in M_{25}^{d}$; на правой половине - линейно возрастающие $\left(\tau_{0}<\tau_{1}\right)$ температурные факторы (9) с $\tau_{0}, \tau_{1} \in T_{15}^{d}$, обозначенные с помощью заглавных букв латинского алфавита от "А” до “J” и от “К” до “Ү”, соответственно.

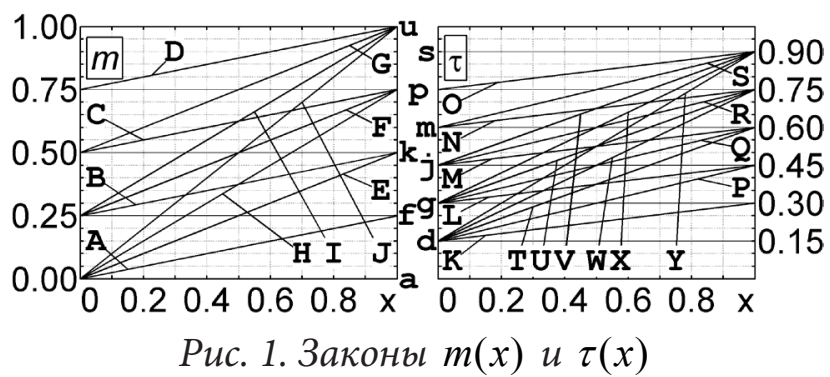

В $[1,2,8,9]$ линейные законы $m(x)$ и $\tau(x)$ обозначались упорядоченными парами строчных букв (элементам множества $M_{05}^{d}$ соответствовали буквы от “a” до “u”, а элементам $T_{05}^{d}$ - буквы от "d" до “s”). Например, закон "B" с $m_{0}=0,25, m_{1}=0,5$ обозначался "fk", а закон "V" с $\tau_{0}=0,45, \tau_{1}=0,9$ обозначался "js".

Результаты вычислительных экспериментов при $\left(m_{1}-m_{0}\right) \in M_{25^{\prime}}^{d}$ и $\left(\tau_{1}-\tau_{0}\right) \in T_{15^{\prime}}^{d}$ для $\theta_{0}, \ldots, \omega_{1}$ представлены на рис. $2-10$, для $q(x)$, 
Анализ влияния тинейно возрастающего вдува и линейно возрастающего температурного ...

$f(x)$ - на рис. 11-19. Распределение случаев сочетания законов управления по рисункам указано в табл. 1.

Таблииа 1

\begin{tabular}{|c|c|c|c|}
\hline $\mid \sigma^{\prime}$ & 0,15 & 0,45 & 0,6 \\
\hline$\left|m^{\prime}\right|$ & K...O & T...V & $\mathrm{W}, \mathrm{X}$ \\
\hline $0,25:$ A...D & рис.2,11 & рис. 3,12 & рис. 4,13 \\
\hline $0,5 \quad$ E...G & рис. 5,14 & рис. 6,15 & рис.7,16 \\
\hline $\begin{array}{l:l}0,75 & \mathrm{H}, \mathrm{I}\end{array}$ & рис. 8,17 & рис. 9,18 & рис. 10,19 \\
\hline
\end{tabular}

\section{2. ВЛИЯНИЕ НА ПАРАМЕТРЫ МАТЕМАТИЧЕСКОЙ МОДЕЛИ}

Утверждение 1. В условиях (2)-(7) для $k \in\{0 ; 1\}$ и любой постоянной $s \equiv C \in S^{c}$ в случае применения (8), (9) для любых $m_{0}, \overline{m_{0}}, m_{1}$, $m_{1} \in M^{c} u \tau_{0}, \tau_{1} \in T^{c}$ если

$$
\underline{m_{0}} \leq \frac{m_{1}}{\tau_{0}} \leq \overline{m_{0}} \leq \overline{m_{1}},
$$

и выполнены условия (9), (10) [8] подчинения параметров:

$$
\begin{aligned}
& \frac{m_{0}}{\leq \overline{m_{0}}}, \\
& \underline{m_{1}} \leq \overline{m_{1}},
\end{aligned}
$$

mo

$$
\begin{aligned}
& \theta_{k}\left(x ; m\left(x ; \underline{m_{0}}, \underline{m_{1}}\right), \tau\left(x ; \tau_{0}, \tau_{1}\right), C\right) \leq \\
& \leq \theta_{k}\left(x ; m\left(x ; \overline{m_{0}}, \overline{m_{1}}\right), \tau\left(x ; \tau_{0}, \tau_{1}\right), C\right), \\
& \omega_{k}\left(x ; m\left(x ; \overline{m_{0}}, m_{1}\right), \tau\left(x ; \tau_{0}, \tau_{1}\right), C\right) \leq \\
& \leq \omega_{k}\left(x ; m\left(x ; \overline{m_{0}}, \overline{m_{1}}\right), \tau\left(x ; \tau_{0}, \tau_{1}\right), C\right)
\end{aligned}
$$

для всех $x \in X$.

Утверждение 2. В условиях (2)-(7) для $k \in\{0 ; 1\}$ и любой постоянной $s \equiv C \in S^{c}$ в случае применения (8), (9) для любых $m_{0}, m_{1} \in M^{c}$ $u \underline{\tau_{0}}, \overline{\tau_{0}}, \underline{\tau_{1}}, \overline{\tau_{1}} \in T^{c}$ если

$$
\begin{gathered}
m_{0} \leq m_{1}, \\
\underline{\tau_{0}} \leq \underline{\tau_{1}}, \overline{\tau_{0}} \leq \overline{\tau_{1}}
\end{gathered}
$$

и выполнены условия (9), (10) [9] подчинения параметров:

$$
\begin{aligned}
& \underline{\tau_{0}} \leq \overline{\tau_{0}}, \\
& \underline{\tau_{1}} \leq \overline{\tau_{1}},
\end{aligned}
$$

mo

$$
\theta_{k}\left(x ; m\left(x ; m_{0}, m_{1}\right), \tau\left(x ; \underline{\tau_{0}}, \underline{\tau_{1}}\right), C\right) \geq
$$

$$
\begin{aligned}
& \geq \theta_{k}\left(x ; m\left(x ; m_{0}, m_{1}\right), \tau\left(x ; \overline{\tau_{0}}, \overline{\tau_{1}}\right), C\right), \\
& \omega_{k}\left(x ; m\left(x ; m_{0}, m_{1}\right), \tau\left(x ; \underline{\tau_{0}}, \overline{\tau_{1}}\right), C\right) \geq \\
& \geq \omega_{k}\left(x ; m\left(x ; m_{0}, m_{1}\right), \tau\left(x ; \overline{\tau_{0}}, \overline{\tau_{1}}\right), C\right)
\end{aligned}
$$

для всех $x \in X$.
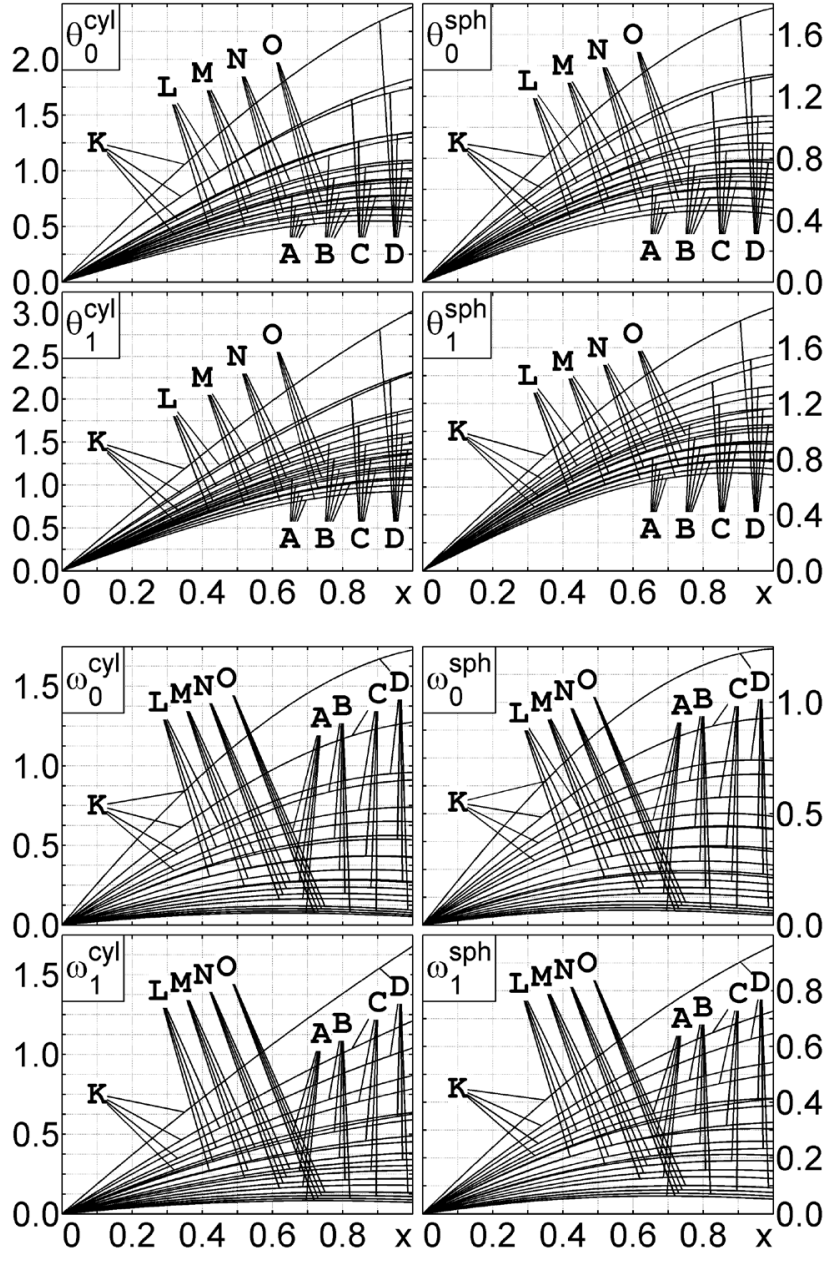

Pис. 2. Зависимости $\theta_{0}(x), \ldots, \omega_{1}(x)$ при

$$
m^{\prime}=+0,25 \text { u } \tau^{\prime}=+0,15
$$

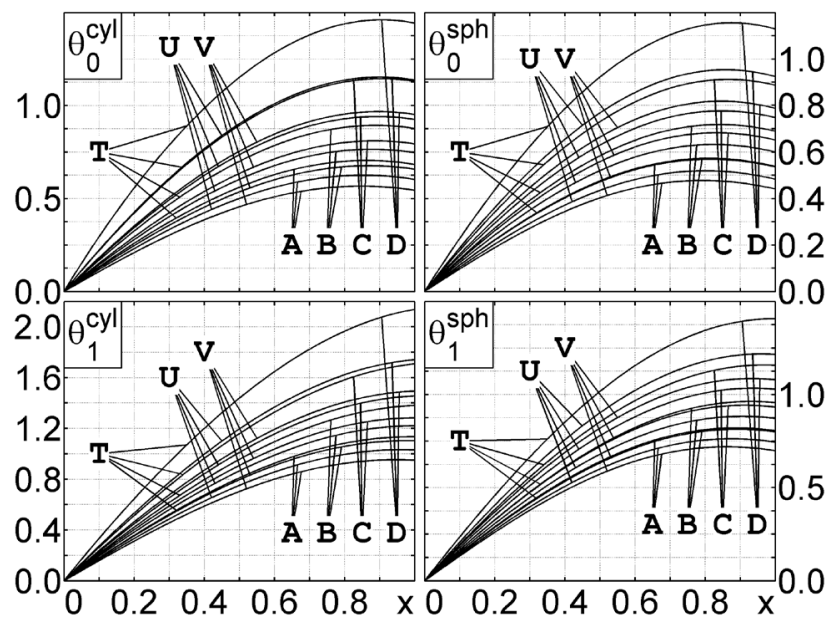




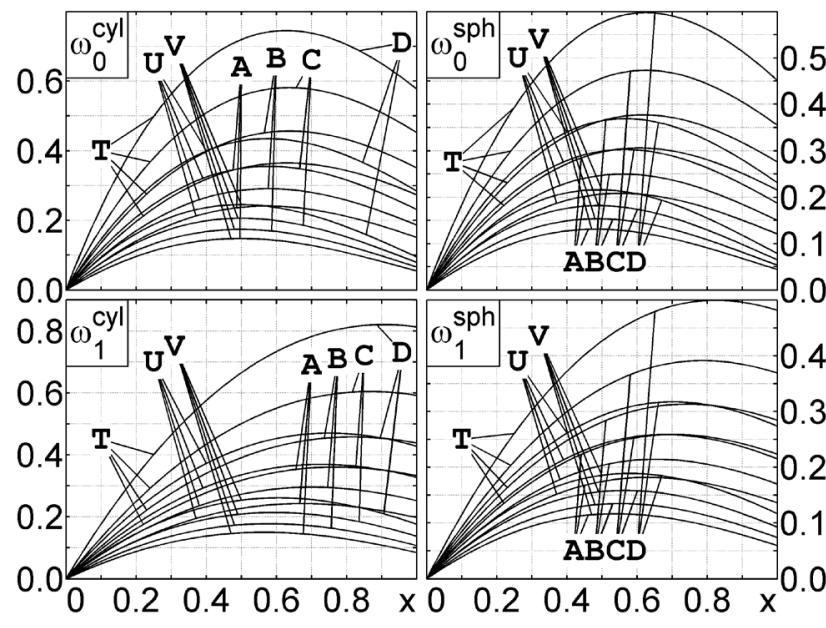

Рис. 3. Зависимости $\theta_{0}(x), \ldots, \omega_{1}(x)$ при $m^{\prime}=+0,25$ u $\tau^{\prime}=+0,45$
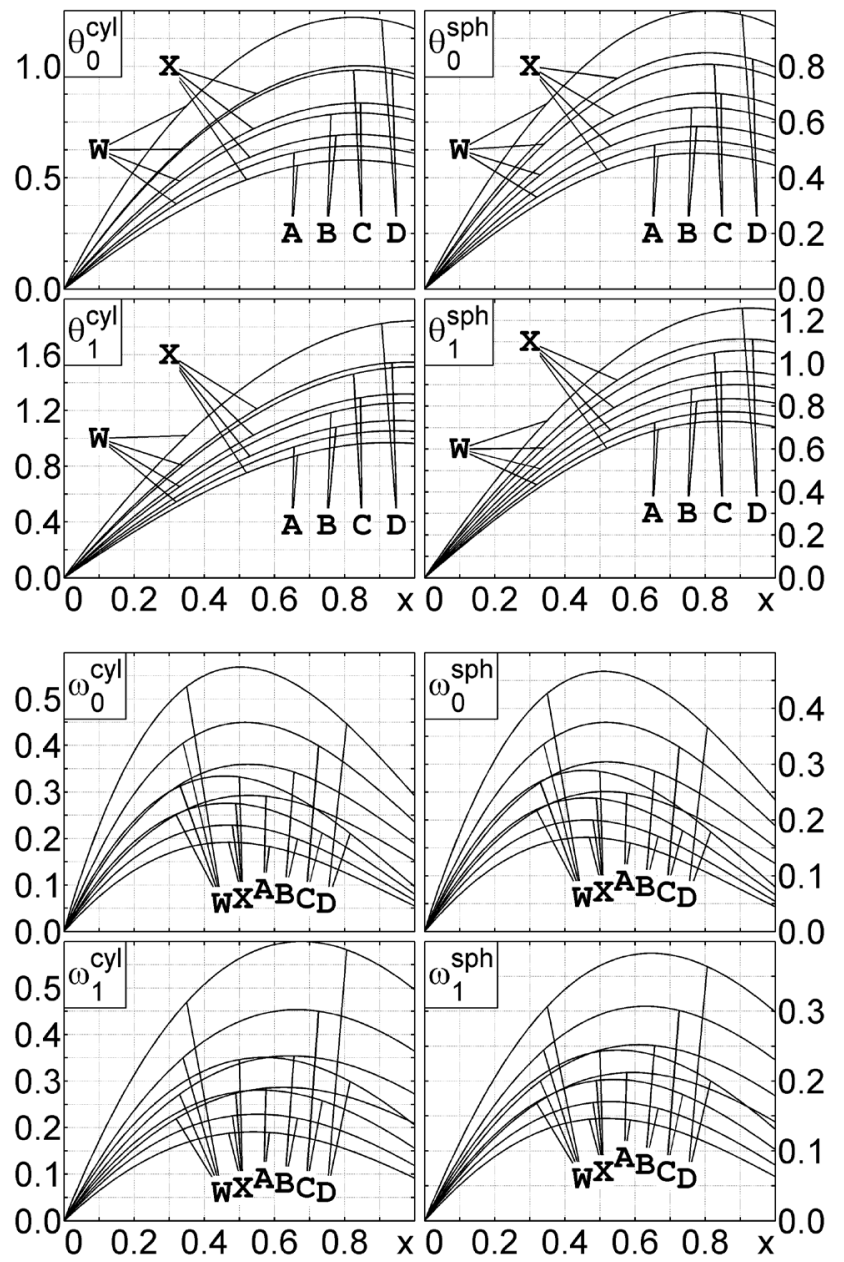

Рис. 4. Зависимости $\theta_{0}(x), \ldots, \omega_{1}(x)$ при $m^{\prime}=+0,25$ u $\tau^{\prime}=+0,6$
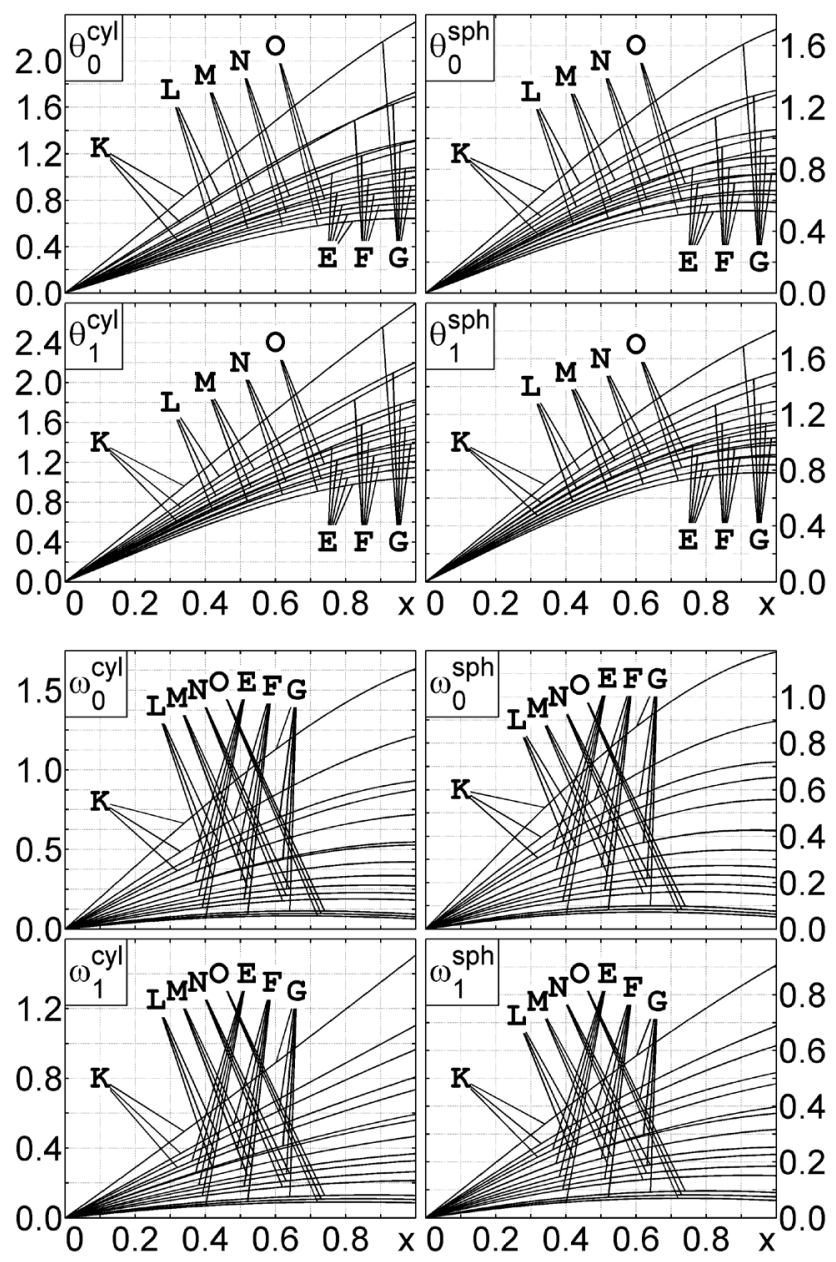

Рис. 5. Зависимости $\theta_{0}(x), \ldots, \omega_{1}(x)$ при $m^{\prime}=+0,5$ u $\tau^{\prime}=+0,15$

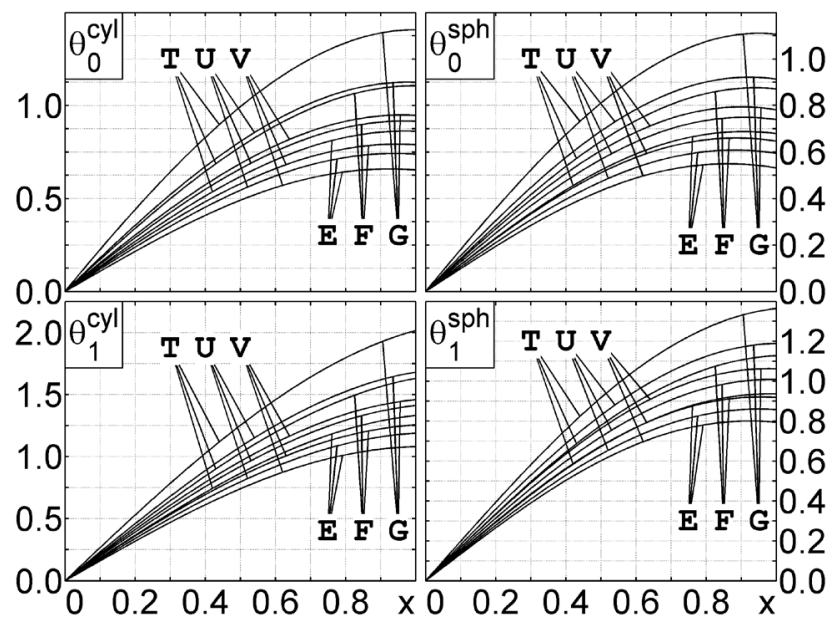


Анализ влияния линейно возрастающего вдува и линейно возрастающего температурного ...

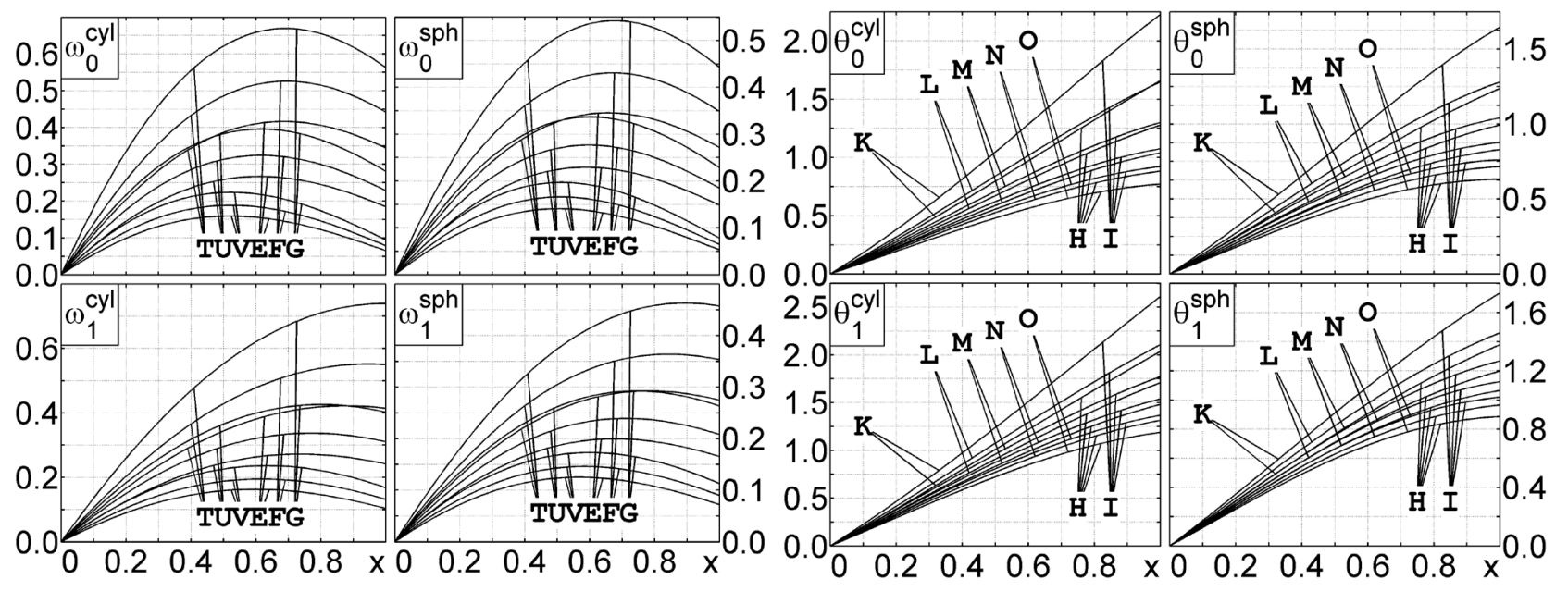

Рис. 6. Зависимости $\theta_{0}(x), \ldots, \omega_{1}(x)$ при $m^{\prime}=+0,5$ u $\tau^{\prime}=+0,45$
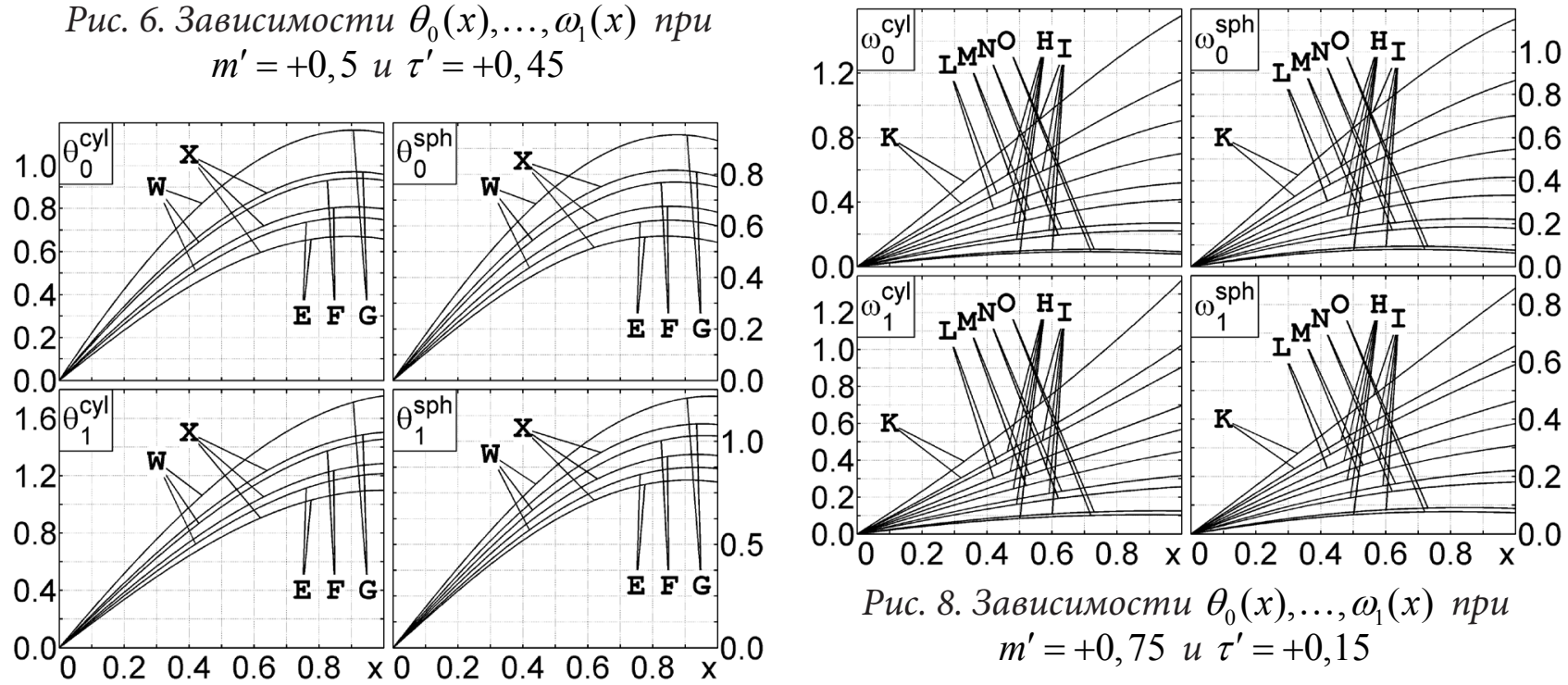

Рис. 8. Зависимости $\theta_{0}(x), \ldots, \omega_{1}(x)$ при $m^{\prime}=+0,75$ u $\tau^{\prime}=+0,15$
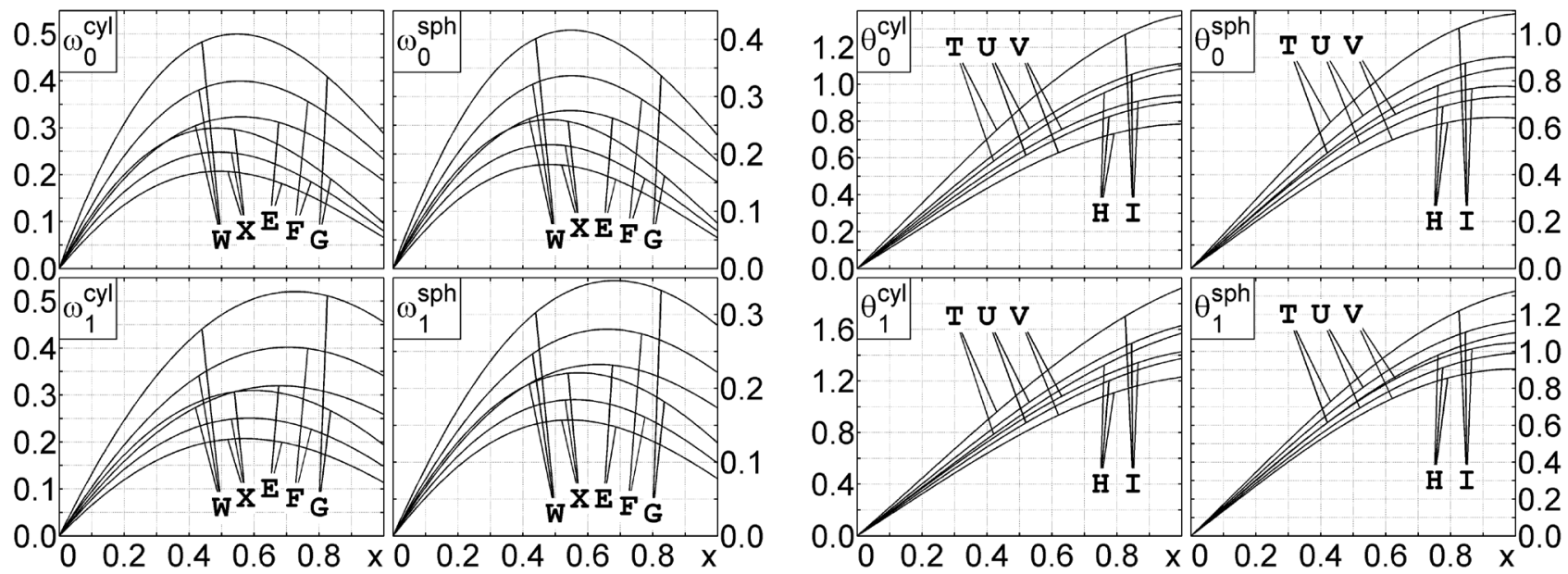

Рuс. 7. Зависимости $\theta_{0}(x), \ldots, \omega_{1}(x)$ при $m^{\prime}=+0,5$ u $\tau^{\prime}=+0,6$ 


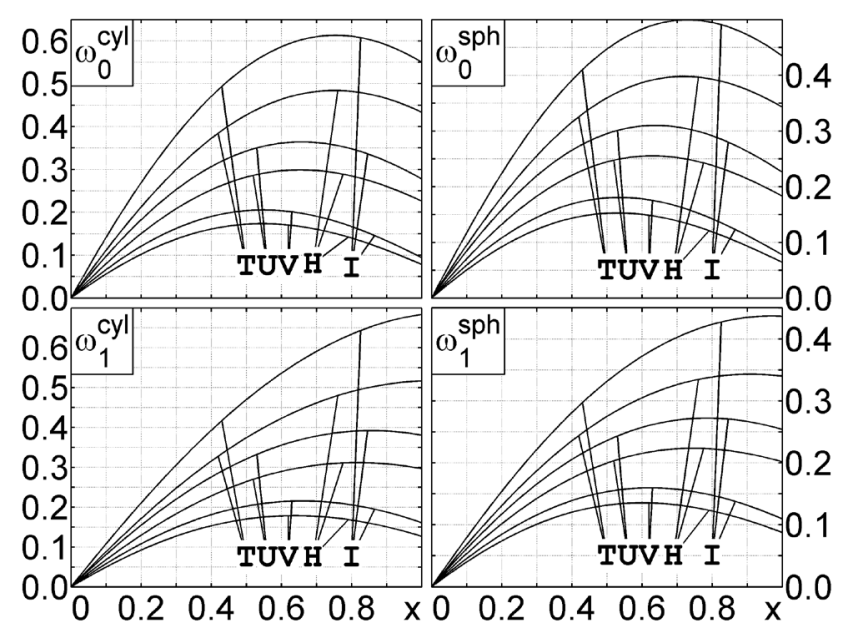

Рuc. 9. Зависимости $\theta_{0}(x), \ldots, \omega_{1}(x)$ при $m^{\prime}=+0,75$ u $\tau^{\prime}=+0,45$
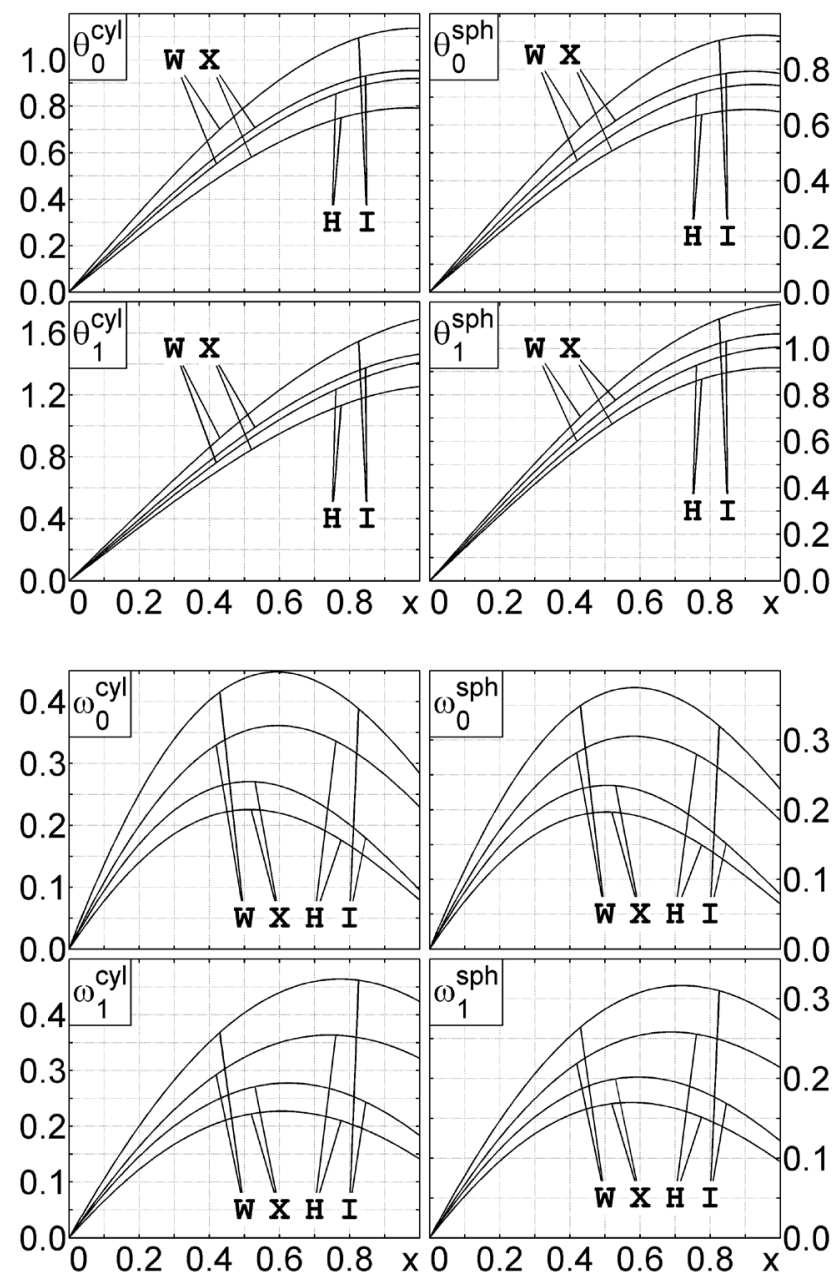

Рис. 10. Зависимости $\theta_{0}(x), \ldots, \omega_{1}(x)$ при $m^{\prime}=+0,75$ u $\tau^{\prime}=+0,6$

\section{3. ВЛИЯНИЕ НА ПАРАМЕТРЫ ТЕПЛОМАССООБМЕНА И ТРЕНИЯ}

Утверждение 3. В условиях (2)-(7) для любой постоянной $s \equiv C \in S_{-}^{c}$ в случае применения (8), (9) для любых $m_{0}, m_{0}, m_{1}, m_{1} \in M^{c} u \tau_{0}$, $\tau_{1} \in T^{c}$ если выполнены условия (16)-(19), то

$$
\begin{aligned}
& f\left(x ; m\left(x ; m_{0}, m_{1}\right), \tau\left(x ; \tau_{0}, \tau_{1}\right), C\right) \geq \\
\geq & f\left(x ; m\left(x ; \overline{m_{0}}, \overline{m_{1}}\right), \tau\left(x ; \tau_{0}, \tau_{1}\right), C\right)
\end{aligned}
$$

для всех $x \in X$.

Утверждение 4. В условиях (2)-(7) для любой постоянной $s \equiv C \in S^{c}$ в случае применения (8), (9) для любъхх $m_{0}, m_{1} \in M^{c} u \tau_{0}, \bar{\tau}_{0}, \tau_{1}$,

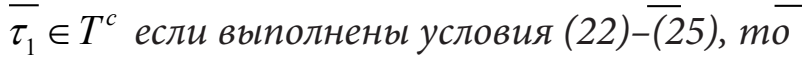

$$
\begin{aligned}
& f\left(x ; m\left(x ; m_{0}, m_{1}\right), \tau\left(x ; \underline{\tau_{0}}, \underline{\tau_{1}}\right), C\right) \leq \\
& \leq f\left(x ; m\left(x ; m_{0}, m_{1}\right), \tau\left(x ; \overline{\tau_{0}}, \overline{\tau_{1}}\right), C\right)
\end{aligned}
$$

для всех $x \in X$.
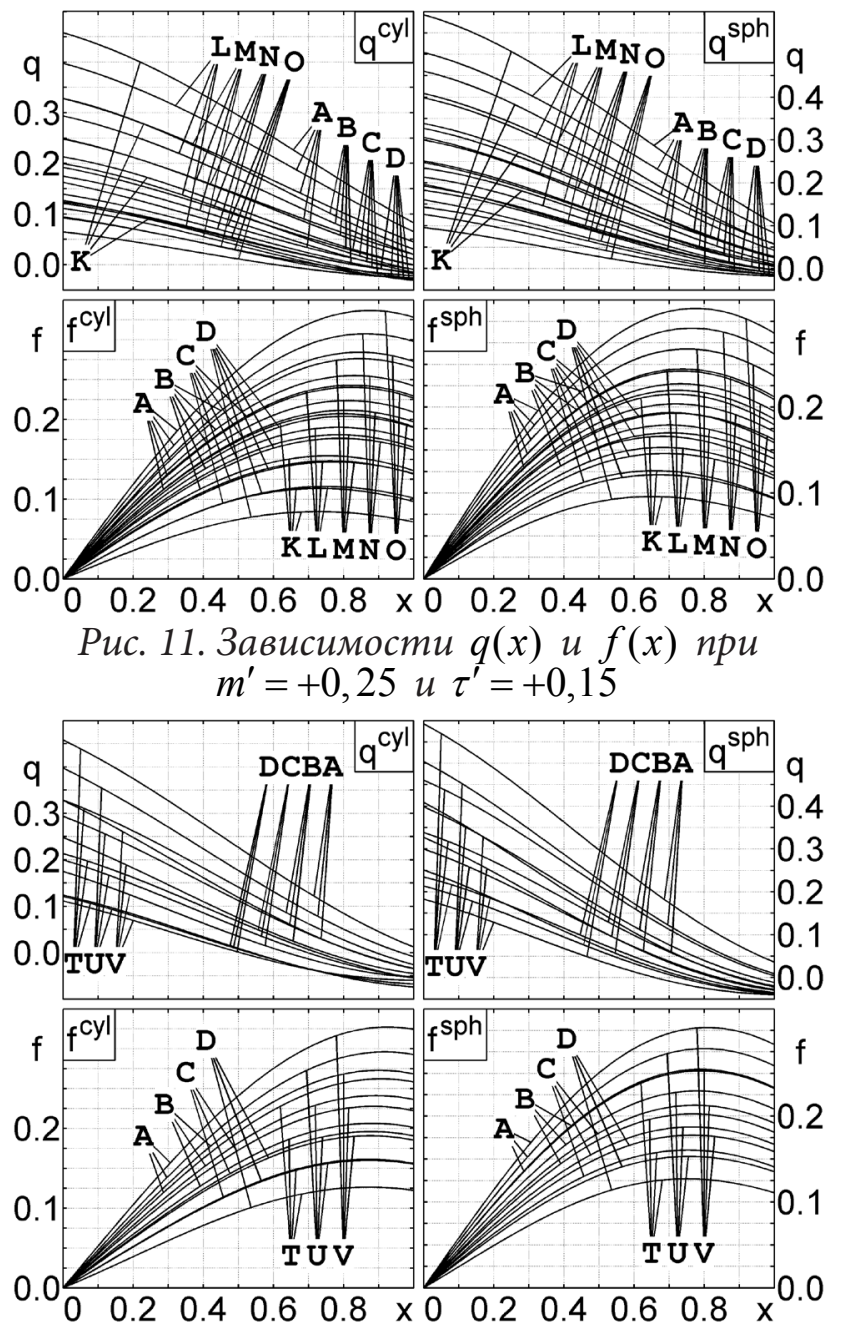

Pис. 12. Зависимости $q(x)$ u $f(x)$ nрu $m^{\prime}=+0,25$ u $\tau^{\prime}=+0,45$ 
Анализ влияния линейно возрастающего вдува и линейно возрастающего температурного ...
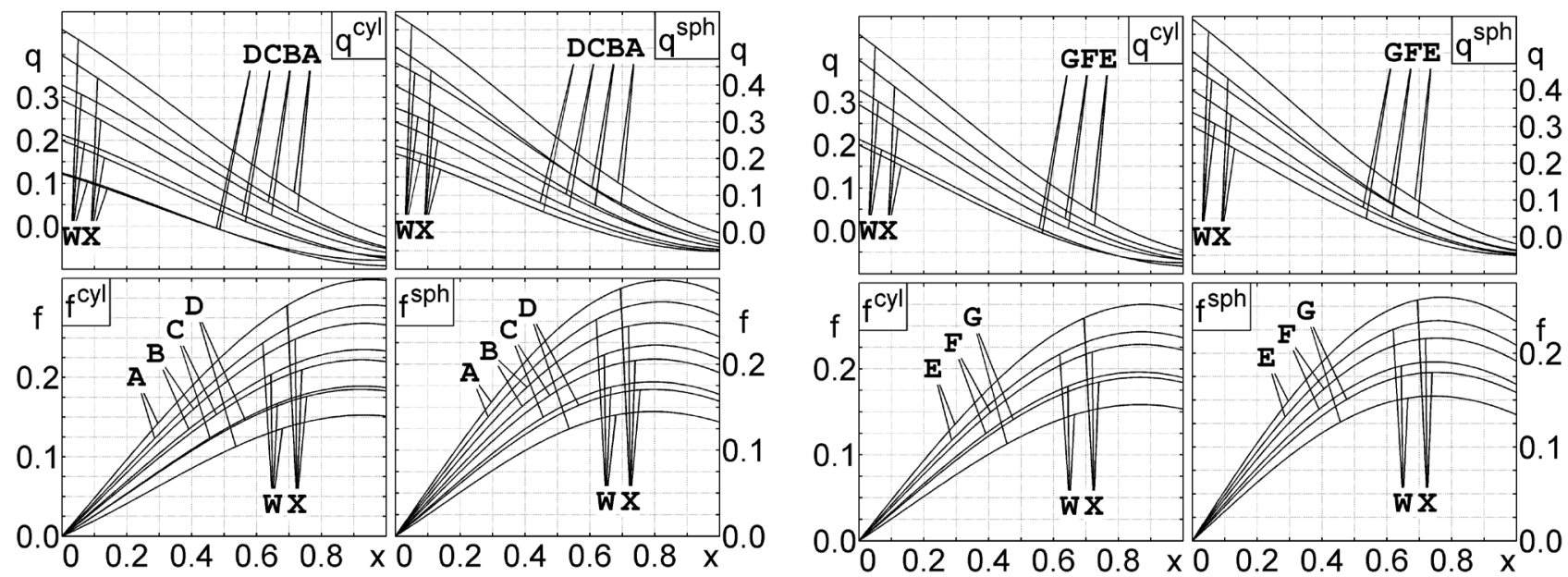

Рис. 13. Зависимости $q(x)$ и $f(x)$ при $m^{\prime}=+0,25$ u $\tau^{\prime}=+0,6$

Рис. 16. Зависимости $q(x)$ и $f(x)$ при $m^{\prime}=+0,5$ u $\tau^{\prime}=+0,6$
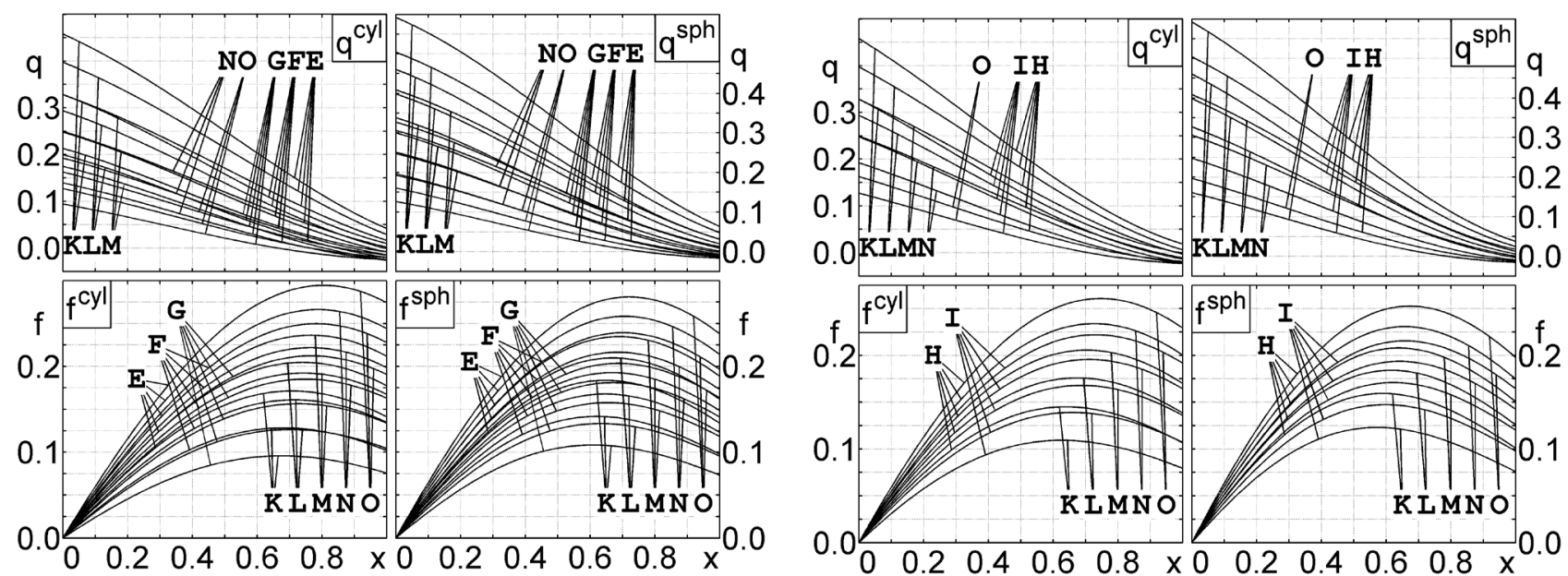

Рис. 14. Зависимости $q(x)$ и $f(x)$ при $m^{\prime}=+0,5$ u $\tau^{\prime}=+0,15$

Рис. 17. Зависимости $q(x)$ u $f(x)$ nри $m^{\prime}=+0,75$ u $\tau^{\prime}=+0,15$
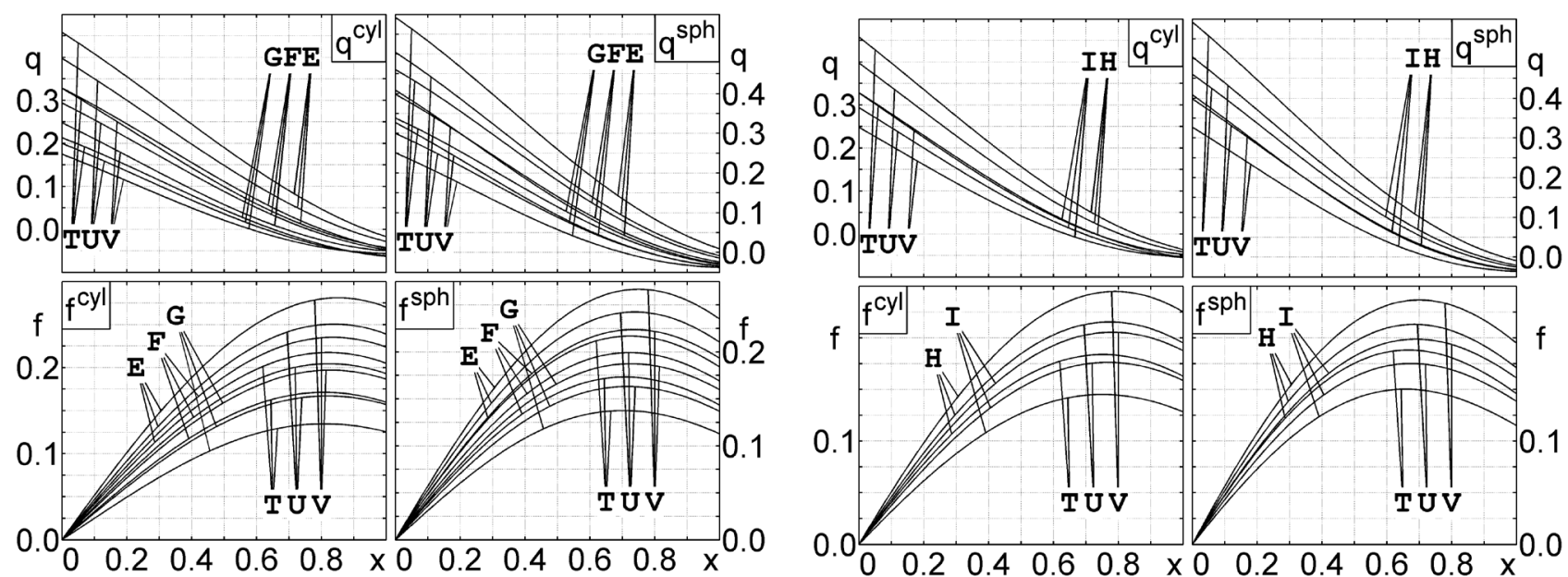

Рис. 15. Зависимости $q(x)$ и $f(x)$ при $m^{\prime}=+0,5 u \tau^{\prime}=+0,45$

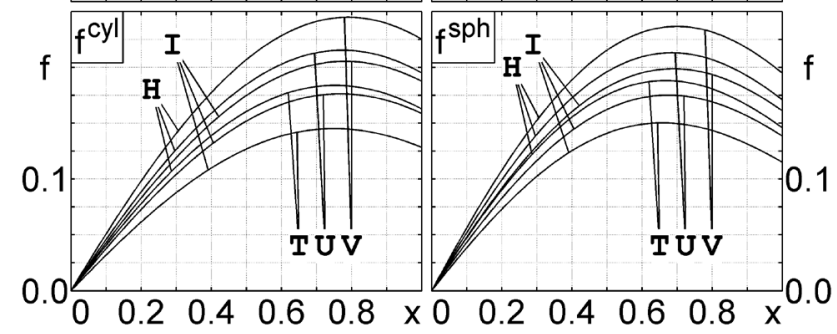

Рис. 18. Зависимости $q(x)$ u $f(x)$ nри $m^{\prime}=+0,75$ u $\tau^{\prime}=+0,45$ 

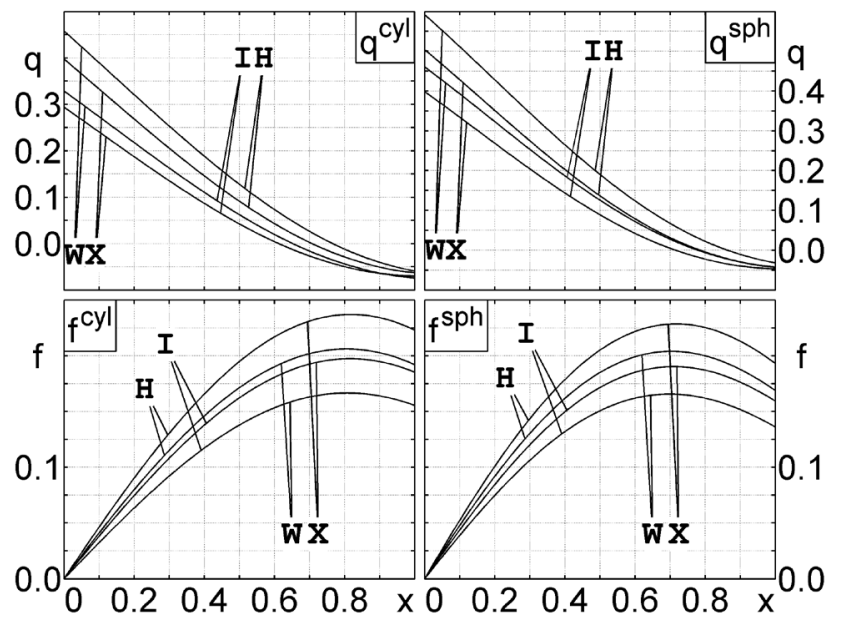

Рис. 19. Зависимости $q(x)$ и $f(x)$ при $m^{\prime}=+0,75$ u $\tau^{\prime}=+0,6$

\section{ЗАКЛЮЧЕНИЕ}

В данной работе изучено влияние на параметры математической модели ламинарного пограничного слоя $\theta_{0}(x), \theta_{1}(x), \omega_{0}(x), \omega_{1}(x)$ и на локальные характеристики тепломассообмена и трения $q(x), f(x)$ одного из сочетаний наиболее простых представителей класса монотонных функций - линейно возрастающего вдува и линейно возрастающего температурного фактора.

Полученные результаты вычислительных экспериментов могут быть использованы в качестве моделей наблюдаемых данных в задачах синтеза эффективного управления как на всём участке [10], так и на его фрагментах [11].

Анализ влияния сочетаний линейных управлений, содержащих убывающие законы, на параметры математической модели ламинарного пограничного слоя и на локальные характеристики тепломассообмена и трения, также, как и анализ влияния сочетания линейно возрастающего вдува и линейно возрастающего температурного фактора на область значений функционалов гиперзвуковой аэродинамики является предметом отдельного исследования.

\section{СПИСОК ЛИТЕРАТУРЫ}

1. Бильченко, Г. Г. О влиянии линейно возрастающего вдува и линейно возрастающего температурного фактора на локальные ха- рактеристики тепломассообмена и трения на проницаемых поверхностях ГЛА / Г. Г. Бильченко, Н. Г. Бильченко // «Некоторые актуальные проблемы современной математики и математического образования. Герценовские чтения-2019». Материалы научной конференции, 8-12 апреля 2019 г. - СПб. : Изд. РГПУ им. А. И. Герцена, 2019. - С. 12-20.

2. Бильченко, Г. Г. О влиянии линейно возрастающего вдува и линейно возрастающего температурного фактора на параметры математической модели на проницаемых поверхностях ГЛА / Г. Г. Бильченко, Н. Г. Бильченко // Современные методы прикладной математики, теории управления и компьютерных технологий: Сборник трудов XII Международной научной конференции «ПМТУКТ-2019», Воронеж, 25-28 сентября 2019 г. - Воронеж: ВГУИТ, 2019. - С. 75-78.

3. Дородницын, А. А. Об одном методе решения уравнений ламинарного пограничного слоя / А. А. Дородницын // Прикладная математика и техническая физика. - 1960. - № 3. C. 111-118.

4. Bilchenko, N. G. On optimum control of laminar boundary layer of electroconductive gas by supersonic flow conditions / N. G. Bilchenko, K. G. Garaev // Proceedings of 12th NATIONAL HEAT TRANSFER CONFERENCE (UIT). L'Aquila, Italy. - 1994. - P. 213-224.

5. Бильченко, Н. Г. Метод А. А. Дородницына в задачах оптимального управления тепломассообменом на проницаемых поверхностях в ламинарном пограничном слое электропроводящего газа / Н. Г. Бильченко // Вестник Воронеж. гос. ун-та. Сер. Системный анализ и информационные технологии.2016. - № 1. - С. 5-14.

6. Бильченко, Н. Г. Вычислительные эксперименты в задачах оптимального управления тепломассообменом на проницаемых поверхностях в ламинарном пограничном слое электропроводящего газа / Н. Г. Бильченко // Вестник Воронеж. гос. ун-та. Сер. Системный анализ и информационные технологии. 2016. - № 3. - C. 5-11.

7. Бильченко, Г. Г. Анализ влияния постоянных управляющих воздействий на область значений функционалов гиперзвуковой аэро- 
Анализ влияния линейно возрастающего вдува и линейно возрастающего температурного ...

динамики / Г. Г. Бильченко, Н. Г. Бильченко // Вестник Воронеж. гос. ун-та. Сер. Системный анализ и информационные технологии. 2018. - № 2. - C. 5-13.

8. Бильченко, Г. Г. Анализ влияния линейного вдува и постоянного температурного фактора на параметры математической модели и локальные характеристики тепломассообмена и трения на проницаемых поверхностях ГЛА / Г. Г. Бильченко, Н. Г. Бильченко // Вестник Воронеж. гос. ун-та. Сер. Системный анализ и информационные технологии. 2019. - № 2. - C. 5-14.

9. Бильченко, Г. Г. Анализ влияния линейного температурного фактора и постоянного вдува на параметры математической модели и локальные характеристики тепломассообмена и трения на проницаемых поверхностях ГЛА / Г. Г. Бильченко, Н. Г. Бильченко // Вест- ник Воронеж. гос. ун-та. Сер. Системный анализ и информационные технологии. - 2019. № 2. - С. 15-22.

10. Бильченко, Г. Г. Обратные задачи тепломассообмена на проницаемых поверхностях гиперзвуковых летательных аппаратов. IV. Классификация задач на всём участке управления / Г. Г. Бильченко, Н. Г. Бильченко // Вестник Воронеж. гос. ун-та. Сер. Системный анализ и информационные технологии. 2018. - № 3. - C. 5-12.

11. Бильченко, Г. Г. Обратные задачи тепломассообмена на проницаемых поверхностях гиперзвуковых летательных аппаратов. V. Смешанные задачи на фрагментах участка управления / Г. Г. Бильченко, Н. Г. Бильченко // Вестник Воронеж. гос. ун-та. Сер. Системный анализ и информационные технологии. - 2018. - № 3. - С. 13-22.

Бильченко Григорий Григорьевич - канд. физ.-мат. наук, научный сотрудник Лаборатории Моделирования Физико-Технических Процессов (при кафедре Теплотехники и Энергетического Машиностроения) Казанского Национального Исследовательского Технического Университета (КНИТУ-КАИ) им. А. Н. Туполева, e-mail: ggbil2@gmail.com

Бильченко Наталья Григорьевна - канд. физ.-мат. наук, научный сотрудник Лаборатории Моделирования Физико-Технических Процессов (при кафедре Теплотехники и Энергетического Машиностроения) Казанского Национального Исследовательского Технического Университета (КНИТУ-КАИ) им. А. Н. Туполева, e-mail: bilchnat@gmail.com 


\title{
ANALYSIS OF THE INFLUENCE OF LINEAR INCREASING BLOWING AND LINEAR INCREASING TEMPERATURE FACTOR ON THE PARAMETERS OF THE MATHEMATICAL MODEL AND LOCAL CHARACTERISTICS OF HEAT AND MASS TRANSFER AND FRICTION ON THE PERMEABLE SURFACES OF THE HYPERSONIC AIRCRAFT
}

\author{
G. G. Bilchenko, N. G. Bilchenko \\ Kazan National Research Technical University (KNRTU-KAI) named after A. N. Tupolev
}

\begin{abstract}
Annotation. The properties of mathematical model of heat and mass transfer and friction control in laminar boundary layer on permeable cylindrical and spherical surfaces of hypersonic aircraft are investigated. The case of application of a combination of linear increasing blowing, linear increasing temperature factor and constant magnetic field for the entire segment of control is considered. The dependences of mathematical model parameters, local heat and mass transfer and friction characteristics on controls are obtained. The computational experiments results are presented. Keywords: control, heat and mass transfer, laminar boundary layer, hypersonic flows, permeable surfaces, linear increasing blowing, linear increasing temperature factor, mathematical model parameters, local heat and mass transfer and friction characteristics.
\end{abstract}

Bilchenko Grigorij Grigorievich - Candidate of Science in Physics and Mathematics, Researcher of Laboratory of Modeling of Physical and Technical Processes, Department of Heat Engineering and Power Engineering Machinery, Kazan National Research Technical University (KNRTU-KAI) named after A. N. Tupolev, e-mail: ggbil2@gmail.com

Bilchenko Natalya Grigorievna - Candidate of Science in Physics and Mathematics, Researcher of Laboratory of Modeling of Physical and Technical Processes, Department of Heat Engineering and Power Engineering Machinery, Kazan National Research Technical University (KNRTU-KAI) named after A. N. Tupolev, e-mail: bilchnat@gmail.com 